\title{
MATRONERÍA, ESTALLIDO SOCIAL Y \\ COVID-19. DISCURSOS DE MATRONAS Y MATRONES CHILENOS EN TORNO AL ROL PROFESIONAL EN CONTEXTOS DE CRISIS
}

Midwifery, Social Outburst and Covid-19. Chilean Midwives' Discourses about their Professional Role in Contexts of Crisis

Augusto Obando-Cid ${ }^{1}$, Gonzalo Infante-Grandón ${ }^{2}$

Autor correspondencia: Augusto Obando-Cid

Correo electrónico: augusto.obando@ufrontera.cl

1. Matrón. Magíster en Ciencias Sociales Aplicadas. Doctor en Procesos Sociales y Políticos en América Latina. Doctor en Ciencias Sociales. Departamento de Salud Pública. Facultad de Medicina, Universidad de La Frontera. (Temuco, Chile)

2. Matrón. Licenciado en matronería. Magíster en Salud Pública Comunitaria y Desarrollo Local. Doctorando en Salud Colectiva. Departamento de Obstetricia y Ginecología. Departamento de Salud Pública. Facultad de Medicina, Universidad de La Frontera. (Temuco, Chile)

Recibido: 11/08/2020 Aceptado: 30/04/2021 


\section{RESUMEN}

INTRODUCCIÓN Y OBJETIVO. La matronería en Chile tiene un incuestionable rol social, con particular protagonismo en contextos de crisis. Nace como profesión, ante una situación sociosanitaria de fenomenal vulnerabilidad de las mujeres. La presente investigación tiene por objetivo analizar los discursos sobre el despliegue e incidencia de la matronería en los escenarios propiciados por el «estallido social» de octubre 2019 y la pandemia de COVID-19. MATERIAL Y MÉTODO. La presente investigación adhiere al paradigma simbólico-interpretativo, desde una metodología cualitativa, situado en el enfoque de estudio de caso, en el que las estrategias de construcción de datos son documentales y conversacionales, utilizándose las técnicas de revisión documental para prensa escrita/online y entrevistas semiestructuradas. Se seleccionó 11 notas de prensa nacional y se realizaron 6 entrevistas semiestructuradas a matronas y matrones que se desempeñan en atención primaria, atención hospitalaria y docencia. Para el análisis de datos se utilizó la perspectiva de Verón de la semiosis social y el análisis de los discursos sociales.

RESULTADOS Y CONCLUSIONES. Frente al estallido social y la crisis sanitaria causada por la COVID-19, la matronería toma un rol activo de denuncia, demanda y emplazamiento a la autoridad sanitaria y gubernamental, visibilizando las necesidades de salud sexual y reproductiva, exigiendo que las gestantes sean consideradas población prioritaria en la pandemia, solicitando protocolos de acción, incluso generando uno, sin obtener respuesta, advirtiendo con interponer acciones legales para proteger a las mujeres y gestantes del país, ante el olvido tácito por parte del Ministerio de Salud.

Palabras clave: matronería; rol profesional; estallido social; COVID-19.

\section{ABSTRACT}

INTRODUCTION AND AIMS. The Midwifery in Chile has an unquestionable social role, with a particular role in crisis contexts. It was born as a profession in the face of a socio-sanitary situation of phenomenal vulnerability of women. This research aims to analyze the discourses on the deployment and incidence of the Midwifery, in the scenarios fostered by the social outbreak of October 2019 and the COVID-19 pandemic. MATERIAL AND METHOD. This research adheres to the interpretive symbolic paradigm, from a qualitative methodology, located in the case study approach, in which the data construction strategies are documentary and conversational, using documentary review techniques for written press -web and semi-structured interviews. 11 national press releases were selected and 6 semi-structured interviews were conducted with midwives who work in Primary Care, Hospital Care and Teaching. For data analysis, Veróns perspective of social semiosis and the analysis of social discourses. RESULTS AND CONCLUSIONS. Faced with the social outbreak and the health crisis caused by COVID-19, the Midwifery takes an active role in denouncing, demanding and summoning the health and government authority. Making sexual and reproductive health needs visible, demanding that pregnant women be considered a priority population in the pandemic, requesting action protocols, even generating one, without obtaining a response, warning with filing legal actions to protect women and pregnant women in the country, before tacit forgetting by the Ministry of Health.

Keywords: Midwifery; Professional Role; Social Outbreak; COVID-19. 


\section{INTRODUCCIÓN}

La matronería en Chile tiene 186 años de historia desde su creación como profesión de la salud en el año 1834 (Binfa et al., 2011; Lillo et al., 2016). El ejercicio profesional se despliega por diversos ámbitos del conocimiento y quehacer sanitario, siendo transversal el acompañamiento de la mujer y su familia a lo largo de todo el ciclo vital. Las matronas y matrones tienen presencia en los diferentes dispositivos de atención sanitaria existentes en Chile, con preeminencia en el ámbito de la sanidad pública.

En el año 1952, en un contexto sanitario y social complejo para el Estado chileno, configurado por una alta mortalidad maternoinfantil, mediante la Ley 10.383, se crea el Servicio Nacional de Salud (SNS) (Giaconi y Valdivia, 1994; Giaconi, 2018). La necesidad de dar amplia cobertura para la atención sanitaria del binomio madre e hijo, en el marco de la generación de una estrategia de salud maternoinfantil, fueron el escenario propicio para que matronas y matrones se constituyeran en actores relevantes, para alcanzar el éxito de las políticas de salud pública de mediados del siglo XX (González y Zárate, 2019), contribuyendo en el mejoramiento de los indicadores de salud, particularmente en lo relacionado con los resultados de salud de la mujer y su familia, en tanto desarrollo de un modelo de «Estado benefactor».

El rol profesional de la matronería se despliega atendiendo a las necesidades, requerimientos y propuestas de las mujeres, no solo en el plano de la salud sexual y salud reproductiva (SSySR), sino también, en la forma de concebir y forjar la sociedad; por ello, la seguridad y el completo bienestar de la mujer constituyen el principal interés de los profesionales Matrona/Matrón (Cerda, 2020).

Lo anteriormente descrito da cuenta del histórico rol social que ha tenido la profesión a lo largo de la historia del país, con particular protagonismo en contextos de crisis. En tanto su creación como carrera profesional universitaria, tiene su génesis en una situación sociosanitaria de fenomenal vulnerabilidad de las mujeres. Es por ello por lo que su concurrencia, en virtud del rol profesional, es de particular interés para la presente investigación, la cual tiene por objetivo analizar el despliegue e incidencia de la matronería en los escenarios propiciados por el «estallido social» de octubre del $2019 \mathrm{y}$ la pandemia de COVID-19 en Chile, desde el discurso de profesionales matronas y matrones pertenecientes al sistema de salud chileno. 


\section{Precarización y desmantelamiento del sistema de salud público como parte de la crisis de los cuidados}

En Chile, hasta los años ochenta, la asistencia sanitaria estaba administrada principalmente por el sector público. El financiamiento era gestionado por el Estado, que brindaba servicios de salud a través de una red de dispositivos de atención médica propia. Alrededor del $90 \%$ de los egresos hospitalarios, y más del $85 \%$ de las atenciones ambulatorias eran efectuados en la infraestructura estatal (Titelman, 2000). Durante la década de los ochenta, con el objetivo de instalar un modelo neoliberal, se llevaron a cabo reformas estructurales del Estado, lo que se tradujo en un cambio de la configuración y funcionamiento del sector de la salud en el país.

En el año 1979 se crea el seguro público de salud, denominado Fondo Nacional de Salud (FONASA), continuador legal del Servicio Médico Nacional de Empleados (SERMENA), dando acceso al ingreso a la modalidad de libre elección (MINSAL, 2016). En el año 1981, en consonancia con los principios y el marco normativo neoliberal — que se buscaba propiciar en la sociedad chilena-, se generó un sistema de salud privado, dando paso a la creación de aseguradoras particulares llamadas Instituciones de Salud Previsional (ISAPRE); esto permitió la generación de un nicho de negocio para el mercado en torno a un derecho básico como la salud, en tanto las y los asegurados tienen, legalmente, gran libertad y amplitud de opciones para optar a diversos tipos de centros asistenciales de salud, sean estos públicos o privados (Gattini, 2018).

Respecto a la municipalización de la Atención Primaria en Salud (APS), la implementación trajo consigo problemas en su financiamiento, afectando la coordinación, referencia y continuidad de la atención entre la APS y los niveles de mayor complejidad de la atención sanitaria (Homedes y Ugalde, 2002). En la actualidad, pese a que la APS es considerada como el eje central de cualquier sistema de salud, dada su amplia cobertura y enfoque de territorialidad, el devenir, en el contexto chileno, está lejos de esta convención (Parada-Lezcano et al., 2016).

Es así como las reformas iniciadas en la década del ochenta generaron una profundización de las desigualdades en salud. Esto ha propiciado un escenario de «claustrofobia territorial» que determina una amplificación de las inequidades sociosanitarias, vigentes hasta el día de hoy (Infante-Grandón, 2017). Estas situaciones, mediadas por la clase/ingreso (capacidad de pago), debieron ser enfrentadas en la década del 2000, con una nueva reforma de salud, conocida como Plan AUGE (Olavarría-Gambi, 
2011), que no tuvo incidencia sobre las inequidades, sino más bien reconfiguró un escenario en el que otros determinantes de la salud como la etnia, el género, relación urbano/rural, entre otras, asumieron protagonismo.

En efecto, el sistema privado, al que se destina el $57 \%$ de los recursos que el país gasta en salud, beneficia a la población con mayores ingresos, más joven y sana. Mientras tanto, el sistema de salud pública, desfinanciado, debe cubrir las necesidades de más de 13 millones de habitantes, de menores ingresos, de mayor edad y, por ende, con mayores factores de riesgo de enfermar (Goyenechea y Sinclaire, 2013). De esta forma, el sistema público de salud se ha visto desmantelado o instrumentalizado por el Gobierno de turno para acrecentar las ganancias de los holdings involucrados en el mercado de la salud (Labra, 2002).

\section{Estallido Social y COVID-19 como atmósferas de interpelación}

El 18 de octubre del año 2019 comienza en Chile el movimiento social más grande de su historia, luego de que estudiantes secundarios, liderados por mujeres, se abalanzaran en masa para ingresar al tren subterráneo sin pagar por el servicio después de un alza de 30 pesos chilenos (0,036 Euros) en el coste del boleto (Silva-Peña y Paz-Maldonado, 2019). La revuelta social congregó rápidamente a todos los sectores de la sociedad de manera transversal. Las demandas dieron cuenta de una profunda desigualdad en el país, cuyo origen y perpetuación se sostienen en el modelo económico-social instalado por la dictadura militar (Ruiz y Caviedes, 2020).

La reducción de la pobreza, el crecimiento económico y la estabilidad democrática (Morán-Faundés, 2019) son los argumentos vinculados a un discurso transversal del espectro político chileno que dan cuenta de un Chile exitoso, que se distancia de sus pares latinoamericanos, refrendado en la calificación del país como un «oasis de estabilidad», en palabras del propio Presidente Piñera, nueve días antes del inicio del «estallido social» (Navarro y Tromben, 2019). Lo anterior, en profunda distancia y contradicción con lo manifestado por las voces sociales que se movilizan en el país, dando cuenta de una larga lista de descontento, que incluyen tópicos centrales — en clave de derechos - como salud, educación, pensiones, agua, fin a la colusión y corrupción empresarial, entre otras, bajo el rótulo de «el pueblo está en la calle pidiendo dignidad» (Silva-Peña y Paz-Maldonado, 2019). 
Las protestas sociales coparon la agenda del año 2019 y llegaron al 2020, constituyendo un momento icónico la Marcha Feminista $8 \mathrm{M}$, que el día 8 de marzo convocó a más de 2 millones de personas en las calles de todo el país (El Mostrador, 2020). Cinco días antes, el Ministerio de Salud (MINSAL) informaba del primer caso de coronavirus en Chile (MINSAL, 2020).

Durante el siglo XX, el mundo se ha visto enfrentado a otras epidemias, tales como las provocadas por la bacteria vibrio cholerae (Cólera), el virus de la inmunodeficiencia humana (VIH/SIDA), el virus del Ébola, el influenza virus subtipo A (H1N1) y el coronavirus en diferentes expresiones (MERS-CoV, SARS-CoV), entre otras. Sin embargo, la aparición del coronavirus tipo 2 (SARS-CoV-2) - mundialmente conocido como COVID-19- en Wuhan (China) (OMS, 2020), se establece como un evento excepcional que ha tensionado y superado los umbrales de lo que hasta ahora se consideraba como posible, tanto a nivel internacional como local. En el caso de Chile, viene a preceder y agudizar la crisis social y política.

La pandemia — que no solo se configura como una entidad biológica, sino que articula dimensiones sociales, económicas, políticas y culturales - interroga a los Estados, no solo a escala de sus sistemas de salud, sino también a niveles individuales, comunitarios y globales. En Chile, dicha interrogación ha dado cuenta de un sistema sanitario débil, fragmentado, golpeado por las consecuencias de un «estallido social» en curso, que pasó de atender una «epidemia de estallidos oculares» (INDH, 2020) a enfrentar una pandemia de coronavirus sin precedentes, con solo días de diferencia. En este trabajo nos planteamos como objetivo analizar el contexto de crisis social y sanitaria que enfrenta la matronería en Chile, desde la perspectiva de matronas y matrones chilenos y del soporte documental publicado en este período.

\section{MATERIAL Y MÉTODO}

La presente investigación adhiere al paradigma simbólico interpretativo (Creswell y Poth, 2018), planteándose que la realidad es construida simbólica e intersubjetivamente por los sujetos, utilizando para ello una metodología cualitativa (Pérez, 1994) que permite el acercamiento a la subjetividad y a las experiencias de matronas y matrones, situándose en el enfoque de estudio de caso (Stake, 2007), dado que se analizará el contexto de crisis social y sanitaria que enfrenta la matronería en Chile. Las estrategias de construcción de 
datos son documentales y conversacionales (Denzin y Lincoln, 2017), utilizándose, para tales fines, las técnicas de revisión documental (para las noticias que se encuentran publicadas en la prensa escrita/online) y las entrevistas semiestructuradas (realizadas a matronas y matrones chilenos).

En consonancia con las estrategias y técnicas documentales propuestas, se construyó un corpus de noticias, de acuerdo al siguiente procedimiento: a) se realizó una búsqueda intencionada de noticias, en los portales de prensa escrita/online, de diarios de circulación nacional, entre los meses de octubre de 2019 y julio de 2020; utilizando las siguientes palabras clave: «Matronas», «Colegio de Matronas», «COVID-19», «Estallido Social», «Salud Sexual y Salud Reproductiva», encontrando 13 noticias, de acuerdo a los criterios descritos; b) luego se efectúo una lectura de título y contenido, bajo los criterios de: relevancia de la noticia, su relación con el objetivo de la presente investigación y que fueran matronas y matrones los principales entrevistados, seleccionando 11 notas de prensa (ver Tabla 1).

Tabla 1. Corpus de Noticias seleccionadas

\begin{tabular}{|l|c|}
\hline \multicolumn{1}{|c|}{ Título de la Noticia } & Medio de prensa \\
\hline $\begin{array}{l}\text { Matronas advierten que clima de crisis social podría } \\
\text { generar partos prematuros (Tele 13, 2019) }\end{array}$ & www.t13.cl \\
\hline $\begin{array}{l}\text { Colegio de Matronas pide al Gobierno que mejore el } \\
\text { Presupuesto de Salud 2020: «Estamos casi con la } \\
\text { misma dotación de la dictadura» (El Mostrador, 2019) }\end{array}$ & www.elmostrador.cl \\
\hline $\begin{array}{l}\text { La razones de la crisis estructural del sistema de salud } \\
\text { público (Rojas, 2019) }\end{array}$ & www.elmostrador.cl \\
\hline $\begin{array}{l}\text { Matronas llaman a participar de manifestaciones por el } \\
\text { Día Internacional de la Mujer (Radio Agricultura.cl, } \\
\text { 2020) }\end{array}$ & www.radioagricultura.cl \\
\hline $\begin{array}{l}\text { Los desafíos de la nueva matronería feminista y } \\
\text { diversa (Rojas, 2020) }\end{array}$ & www.elmostrador.cl \\
\hline $\begin{array}{l}\text { Seis Matronas del Sótero del Río con COVID-19 tras } \\
\text { contagio de funcionaria en culto evangélico } \\
\text { (Cooperativa.cl, 2020) }\end{array}$ & www.cooperativa.cl \\
\hline $\begin{array}{l}\text { Matronas piden al Gobierno no olvidar situación de } \\
\text { embarazadas durante la pandemia (Casas, 2020) }\end{array}$ & www.biobiochile.cl \\
\hline
\end{tabular}




\begin{tabular}{|l|c|}
\hline $\begin{array}{l}\text { Colegio de Matronas pidió que el Gobierno apoye la } \\
\text { extensión del postnatal (The Times Chile, 2020) }\end{array}$ & thetimes.cl \\
\hline $\begin{array}{l}\text { Colegio de Matronas pidió al Gobierno firmar } \\
\text { protocolo de acción para el cuidado de los profesionales } \\
\text { y las mujeres (Castillo, 2020) }\end{array}$ & www.adnradio.cl \\
\hline $\begin{array}{l}\text { Colegio de Matronas: «Embarazadas no son } \\
\text { consideradas población de riesgo en la pandemia» (El }\end{array}$ & www.eldesconcierto.cl \\
$\begin{array}{l}\text { Desconcierto, 2020) } \\
\text { Matronas critican al Gobierno por primer fallecimiento } \\
\text { de puérpera por COVID-19 (El Periodista Online, }\end{array}$ & www.elperiodista.cl \\
2020) & \\
\hline
\end{tabular}

Fuente: Elaboración propia

Con relación a las matronas y matrones participantes, fueron invitadas/invitados (Martínez-Salgado, 2012), recogiendo su aceptación vía correo electrónico, considerando su deseo de participar, respetando cabalmente sus intenciones, temores o rechazos (Marzano, 2007); se seleccionaron por conveniencia, considerando la posibilidad y facilidad de acceder a ellas/ellos (Martínez-Salgado, 2012), dado que, en el momento de realizar las entrevistas, la pandemia de COVID-19 se superponía al "estallido social", requiriendo un intenso despliegue de matronas y matrones en el sistema sanitario chileno, lo que implicó que algunas(os) de ellas(os) se manifestarán imposibilitadas(os) de acoger favorablemente la invitación, excusándose de participar; los criterios de inclusión fueron que las matronas y matrones se encontraran laboralmente activos, con experiencia laboral en el ámbito de la sanidad pública. Además, se buscó la diversidad de perfiles, dado que las y los informantes se ocupan en distintos niveles de atención del sistema de salud, con diferentes ámbitos de desempeño; con el mismo objetivo, los años de antigüedad en la profesión y la estancia en el cargo son diversos, lo que se tradujo en la selección de 6 informantes clave, como se puede ver en la Tabla 2. 
Tabla 2. Caracterización de informantes claves

\begin{tabular}{|c|c|c|c|c|c|c|}
\hline Informante & Sexo & $\begin{array}{l}\text { Edad } \\
\text { (Años) }\end{array}$ & $\begin{array}{l}\text { Nivel de } \\
\text { atención }\end{array}$ & $\begin{array}{l}\text { Ámbito de } \\
\text { Desempeño }\end{array}$ & $\begin{array}{l}\text { Estancia } \\
\text { en el } \\
\text { cargo }\end{array}$ & $\begin{array}{l}\text { Años } \\
\text { de } \\
\text { egreso }\end{array}$ \\
\hline Matrón 1 & Masculino & 41 & $\begin{array}{l}\text { II y III nivel } \\
\text { de atención }\end{array}$ & $\begin{array}{l}\text { Docencia } \\
\text { Universitaria }\end{array}$ & 6 años & 17 \\
\hline Matrona 2 & Femenino & 54 & $\begin{array}{l}\text { I nivel de } \\
\text { atención } \\
\text { APS }\end{array}$ & $\begin{array}{l}\text { Clínico y Directivo. } \\
\text { Matrona Jefa de } \\
\text { servicio y } \\
\text { coordinadora del } \\
\text { estamento en la APS } \\
\text { comunal }\end{array}$ & 10 años & 30 \\
\hline Matrón 3 & Masculino & 32 & $\begin{array}{l}\text { I nivel de } \\
\text { atención } \\
\text { APS }\end{array}$ & $\begin{array}{l}\text { Clínico y Directivo. } \\
\text { Jefatura del Programa } \\
\text { de Salud la Mujer }\end{array}$ & 8 años & 8 \\
\hline Matrona 4 & Femenino & 44 & $\begin{array}{l}\text { I nivel de } \\
\text { atención } \\
\text { APS }\end{array}$ & $\begin{array}{l}\text { Clínico y Directivo. } \\
\text { Jefatura del Programa } \\
\text { de Salud la Mujer }\end{array}$ & 16 años & 21 \\
\hline Matrona 5 & Femenino & 37 & $\begin{array}{l}\text { III nivel de } \\
\text { atención }\end{array}$ & Clínico & 8 años & 13 \\
\hline Matrona 6 & Femenino & 67 & $\begin{array}{l}\text { II y III nivel } \\
\text { de atención }\end{array}$ & $\begin{array}{l}\text { Docencia } \\
\text { Universitaria }\end{array}$ & 28 años & 45 \\
\hline
\end{tabular}

Fuente: Elaboración propia

Las entrevistas fueron de carácter semiestructurado, basándose en un guion de preguntas, enviadas previamente a través de correo electrónico, junto al consentimiento informado, realizándose a través de las plataformas virtuales Meet y Zoom, grabadas y transcritas.

Para el análisis de datos se utilizó la perspectiva de Verón (1993; 2013) sobre la semiosis social y la producción de los discursos sociales, lo cual se justifica 
epistemológica y metodológicamente, en tanto el objeto de estudio son los discursos de matronas y matrones acerca del «estallido social» y la pandemia de COVID-19; ambos contextos de crisis se entrecruzan, constituyendo el objeto de análisis de la presente investigación. Este discurso posee «condiciones de producción», generando su propia «gramática de producción» (Verón, 2013).

El «estallido social» y la pandemia del COVID-19 son fenómenos contingentes y complejos, que se encuentran actualmente en desarrollo, razón por la cual se consideró relevante nutrir la presente investigación tanto con los discursos de la matronería presentes en los medios de comunicación como con los discursos de matronas y matrones desde su propia subjetividad situada, ya sea en la atención clínica de APS como en la atención hospitalaria y la labor docente, con el fin de explorar los discursos y los sentidos que emergen en torno a la matronería y los contextos de crisis analizados.

El procedimiento analítico, para lograr significación, se realizó mediante dos estrategias: 1) la interpretación directa de unidades textuales, distinguiéndolas, extrayéndolas y seleccionándolas del corpus de noticias y de las entrevistas, entendiéndose que ambos corpus poseen diferentes «gramáticas de producción» (Verón, 2013), integrándolos posteriormente para la construcción de sentido general entre ellos; 2) la suma y comparación de las unidades textuales, hasta poder decir algo de ellas como conjunto; 3) finalmente se clasificaron en códigos y categorías, siendo organizadas en redes de análisis, lo que permitió llegar a conclusiones que dan respuesta al objetivo de la presente investigación. Para el manejo de los datos cualitativos se utilizó el software ATLAS.ti en su última versión.

\section{Aspectos Éticos}

En relación a los aspectos éticos y legales; basados en la Ley 20.120 (2006) sobre la investigación científica en el ser humano, su genoma y que prohíbe la clonación humana, y la Ley 20.584 (2012), que regula los derechos y deberes que tienen las personas en relación con acciones vinculadas a su atención en salud, especialmente en aquellos aspectos referentes a la solicitud de consentimiento informado, la protección de la identidad y la clasificación del estudio; de acuerdo con sus implicaciones para con la indemnidad de los sujetos participantes, se plantea que la presente investigación no vulnera su integridad, dado que la participación fue voluntaria, previa solicitud de consentimiento informado firmado vía virtual y remitido por correo electrónico, 
protegiendo la identidad de entrevistadas y entrevistados, no recabando datos sensibles de los participantes, por lo cual es considerada de bajo riesgo.

\section{RESULTADOS Y DISCUSIÓN}

Considerando el corpus de prensa, en específico las noticias: «Colegio de Matronas pide al Gobierno que mejore el Presupuesto de Salud 2020: "Estamos casi con la misma dotación de la dictadura"» (El Mostrador, 2019) y «Las razones de la crisis estructural del sistema de salud público» (Rojas, 2019), se describe el escenario y contexto para el estallido social desde la perspectiva de salud y de la matronería.

Unidades textuales, como las que a continuación se presentan, permitieron construir los códigos y categorías presentes en la red de análisis de la Figura 1:

No es posible que todavía tengamos prácticamente la misma dotación en los servicios públicos desde la dictadura de Pinochet. Necesitamos una inyección de recursos para la salud pública que sea sustancial y acorde a los requerimientos de la población más vulnerable. (Anita Román, presidenta del Colegio de Matronas y Matrones, El Mostrador, 2019)

La crisis a la que ha llegado nuestro sistema de salud tiene su origen en las reformas establecidas en los años $80^{\prime}$, en plena dictadura, como también por las políticas que profundizaron un modelo que negocia con las enfermedades de la gente. (Katiuska Rojas, presidenta del Colegio de Matronas y Matrones Regional Santiago, 2019)

Con todas estas evidencias, la población chilena ya no quiere seguir esperando más soluciones a largo plazo. El Estado debe jugar inmediatamente un rol de aseguramiento de las condiciones sociales y económicas que logren disminuir las brechas de inequidad en salud, cambiando las lógicas de mercado y fortaleciendo al sector público con un enfoque de derechos, y para esto necesitamos urgente una reforma estructural de salud. (Katiuska Rojas, presidenta del Colegio de Matronas y Matrones Regional Santiago, 2019) 
Figura 1. Estallido social y su relación con la crisis de salud en Chile, desde la perspectiva de la matronería

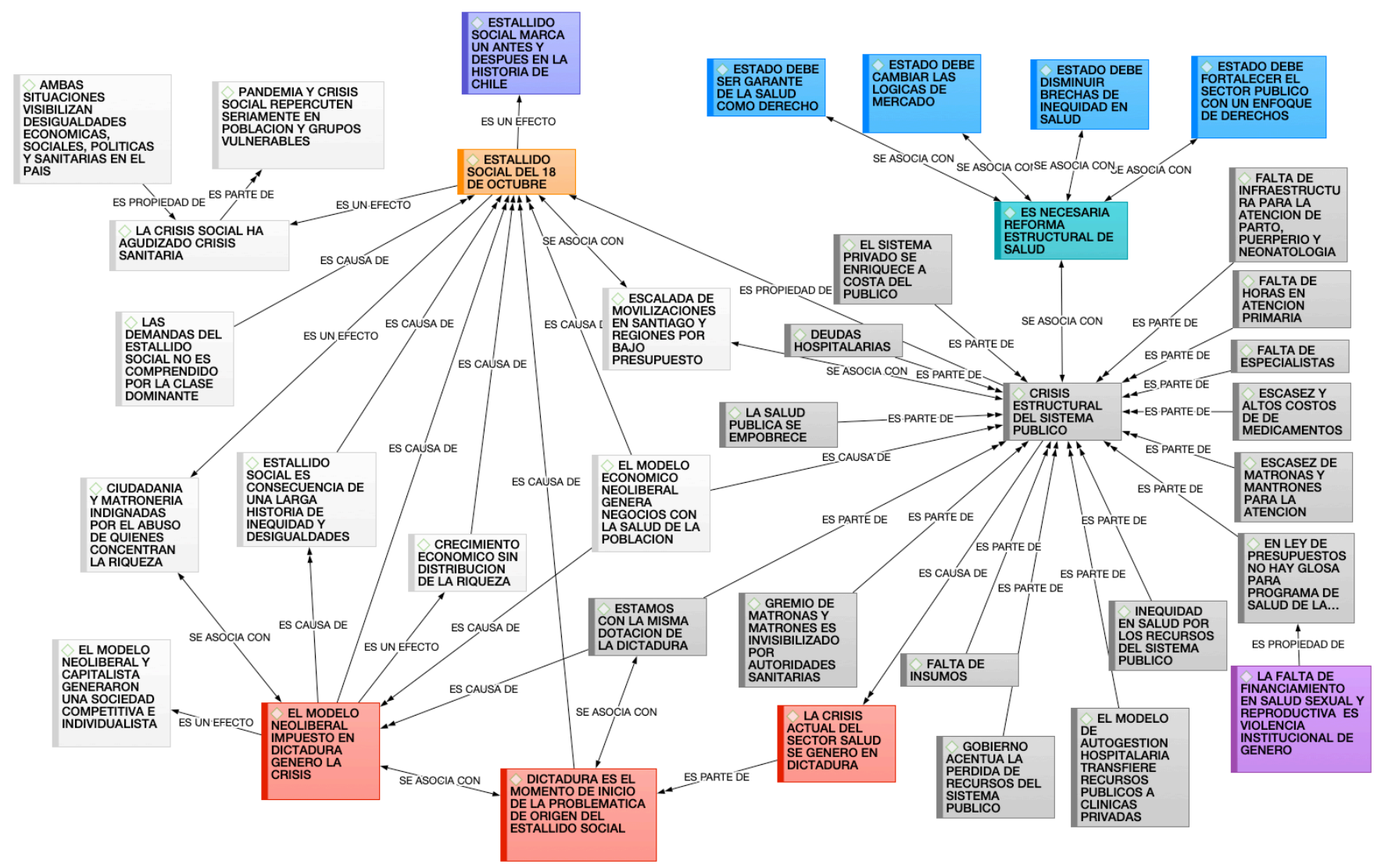

Fuente: Elaboración propia

Según se observa en la Figura 1, la instalación del modelo neoliberal — durante la dictadura-, emerge como causa del «estallido social» del 18 de octubre en Chile. En este sentido, al modelo se le atribuye la generación de un gran crecimiento económico, acompañado por gran desigualdad en la distribución del ingreso en la población, como lo señalan Morán-Faundés (2019), Navarro y Tromben (2019) y Ruiz y Caviedes (2020). En paralelo, tuvo lugar el ingreso del sector salud al mercado, propiciando la participación de actores privados, lo cual culmina con la crisis estructural del sistema público de salud, como lo aseveran Titelman (2000) y Gattini (2018), lo que, desde el discurso de la dirigencia del Colegio de Matronas y Matrones, es parte de las causas del «estallido social».

Se describe, claramente, que la crisis social precede a la crisis sanitaria, transitando hacia la agudización con la pandemia de COVID-19; ambas crisis repercuten en la población general y en los grupos vulnerables de la sociedad, develando desigualdades económicas, sociales, políticas y sanitarias existentes en el país, en coherencia con lo planteado por Silva-Peña y Paz-Maldonado (2019) y Fuentes-García (2020). 
La crisis sanitaria, que es parte de la crisis social, se observa a través de este empobrecimiento histórico del sector público, generado por las lógicas del mercado, siguiendo el análisis de Labra (2002). La desigualdad en la asignación de recursos, la fuga de dineros hacia los centros de atención privada — favorecido por los diferentes gobiernos a través de reformas-, generan una enorme desigualdad económica entre ambos sistemas, lo que da lugar a inequidades en salud entre quienes son atendidos por el sistema público, en contraposición con quienes concurren al sistema privado, en concordancia con lo planteado por Castillo y Molina (2020).

La carencia de recursos se expresa en la deuda hospitalaria, en la falta de especialistas, de infraestructura, de insumos y de medicamentos, sumado al alto costo de estos últimos en el mercado. La falta de horas en APS y la escasez de matronas y matrones para la atención sanitaria, en un contexto en el que el presupuesto para el sector disminuye, es lo que generó las movilizaciones de todos los gremios de la salud. Por otro lado, se menciona que no existe glosa para la SSySR en el presupuesto, situación que fue denunciada por el Colegio de Matronas y Matrones, considerando que, esta particular falta de financiamiento, es violencia estructural de género (Rojas, 2019).

Figura 2. Atención en Salud Sexual y Salud Reproductiva, crisis social y sanitaria en Chile, desde la perspectiva de la matronería

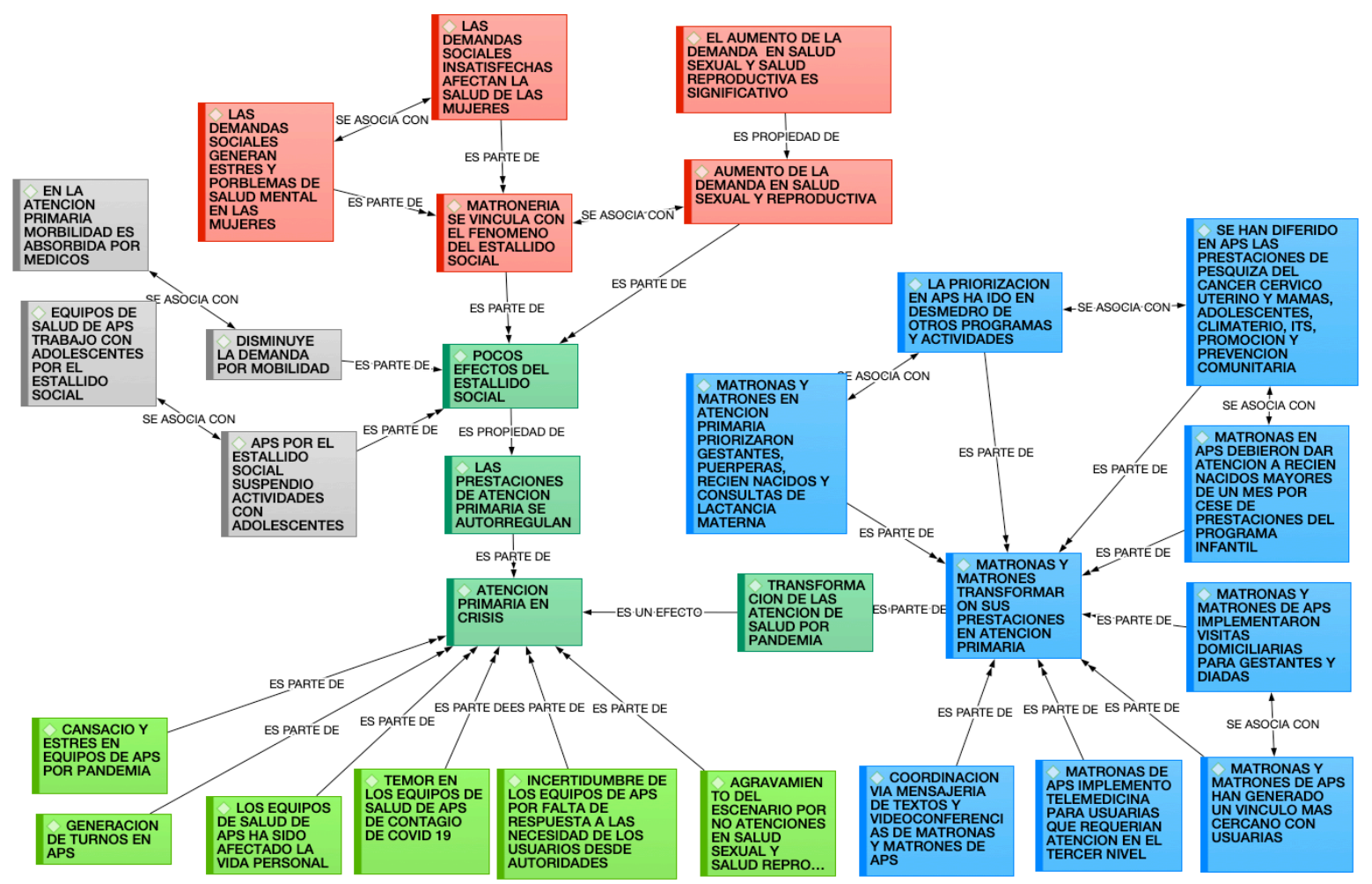

Fuente: Elaboración propia 
La red de análisis de la Figura 2 se construye desde los enunciados presentes en los corpus de las entrevistas realizadas a matronas y matrones, dando cuenta del accionar profesional en el ámbito de la SSySR, en los contextos de crisis sanitaria y social.

Según el discurso de las matronas y matrones, la APS no vio tan afectadas sus funciones en relación con el «estallido social»; sin embargo, el fenómeno de autorregulación que posee este nivel de atención hizo disminuir las consultas de morbilidad y el trabajo con grupos sociales de la comunidad, entre ellos con las y los adolescentes; por otro lado, generó un aumento de las necesidades en SSySR, que, a juicio de las matronas y matrones, no se suspendieron, sino que continúan presentándose como demandas de salud de las mujeres.

Por otra parte, este vínculo de matronas y matrones con las mujeres —usuarias del sistema - les permite suscribir a las demandas sociales, además de observar el estrés y las problemáticas de salud mental que emergen a posterior del «estallido social» ante la ausencia de respuestas por parte del Estado, en correlato con lo planteado por FuentesGarcía (2020).

Unidades textuales, como las que a continuación se presentan, permitieron construir los códigos y categorías presentes en la red de análisis de la Figura 2.

Dejo de manifiesto que la demanda en prestaciones en APS, se auto regula por sí misma. Es decir, disminuye en gran medida la demanda de atenciones por morbilidad y otras, sin embargo, las prestaciones desde el punto de vista de la salud sexual y reproductiva se mantienen en un nivel de demanda significativo. (M. Entrevistad* 5)

También se hizo evidente el stress que provoca en las mujeres las demandas sociales no satisfechas y que dado la confianza y cercanía que la usuaria tiene con la matrona, lo dan a conocer al profesional en una forma de consulta de salud mental, obligando a realizar intervención en crisis en muchos de estos casos. (M. Entrevistad*6)

Lo complejo para la APS es la crisis sanitaria generada por la pandemia, que ha tenido diversos efectos sobre los equipos de salud, tales como: el cansancio y estrés, la generación de turnos para otorgar las prestaciones, el temor ante el contagio por COVID19, la incertidumbre por la ausencia de respuestas por parte de las autoridades, en coherencia con el analisis de contexto realizado por Giovanella et al. (2021); presagiando un agravamiento del escenario, debido a la desatención de necesidades en SSySR de la población, desde la perspectiva de matronas y matrones. 
Lo relevante del relato de matronas y matrones es que debieron transformar profundamente sus prestaciones y las formas de otorgarlas en APS, a diferencia de otros niveles de atención. Se destaca la priorización de algunas actividades y programas por encima de otros, tal es el caso del control prenatal, la atención de la puérpera y del recién nacido, además de la atención en planificación familiar y regulación de la fecundidad, desarrolladas a través de la estrategia de visita domiciliaria integral, en correlato con lo descrito por Infante y Obando (2020). Sin embargo, otros programas fueron dejados de lado, entre ellos la pesquisa de cáncer cérvico-uterino y cáncer de mamas, la atención de adolescentes, climaterio e infecciones de transmisión sexual (ITS), además de todas las acciones de promoción y prevención que se realizan con grupos organizados de la comunidad, sobre la base de una atención por protocolo «más distante», de acuerdo a lo planteado por López (2020).

En relación con el trabajo administrativo y de gestión, este se coordinó a través del teletrabajo, utilizando tecnologías de la información y la comunicación, como mensajería de texto y videoconferencias. Por otra parte, y en esa misma línea, el uso de la telemedicina, para otorgar prestaciones, se ha transformado en una herramienta fundamental en este escenario de pandemia, destacando experiencias como la de la maternidad del Hospital Dra. Eloísa Díaz, de la comuna de La Florida en la Región Metropolitana, que inclusive pavimentó el uso de redes sociales como Instagram para relacionarse más ágilmente con sus usuarias (Leiva et al., 2021).

Se presentan a continuación unidades textuales que dan cuenta de los códigos y categorías de la red de análisis en el ámbito de la gestión:

Creo que, una vez más, Matronas y Matrones, asumen un rol profesional comprometido con la salud de la población que atienden, trabajo silencioso, adaptándose a la realidad de cada lugar. Incorporando acciones muy necesarias, por ejemplo: Control Prenatal en domicilio, pero como siempre, en una especie de «primera línea en la sombra». (M. Entrevistad*3)

Desde mediados de marzo del año en curso, el estamento completo de Matronas y Matrones del Departamento de Salud Municipal (DSM) tuvo que adecuar sus actividades debido a la contingencia actual en la APS, se coordinaron turnos de atención, se habilitaron líneas telefónicas y mensajería de texto para realizar teleconsultas, se disminuyó la atención presencial solo a gestantes, se mantuvo el recambio de métodos anticonceptivos (MAC) y atenciones díadas a domicilio, lo que en parte nos ha llevado a reforzar la importancia de la Salud Familiar, ya que hemos ido conociendo de mayor 
manera las diferentes realidades de las familias inscritas en el DSM. (M. Entrevistad* 1)

Las atenciones se priorizaron en las gestantes, puérperas y recién nacidos y consultas de lactancia materna. Además de atenciones en planificación familiar ya sean por teleconsulta o en casos muy necesarios presenciales. (M. Entrevistad* 2)

No puedo dejar de mencionar que los lineamientos que vienen desde el MINSAL, no se acercan en lo más mínimo a la realidad local; lo mismo ocurre con las directrices del Servicio de Salud, que llega a tal punto que no hay concordancia entre sí, entre los diferentes subdepartamentos. (M. Entrevistad*4)

La Figura 3 representa la red de análisis que describe el accionar del Colegio de Matronas y Matrones. Según lo atestigua el corpus de prensa revisado, este gremio ha tenido un rol social y político preponderante, tanto en el «estallido social» como durante la pandemia del COVID-19.

Figura 3. Acciones del Colegio de Matronas y Matrones frente a la crisis social y sanitaria en Chile

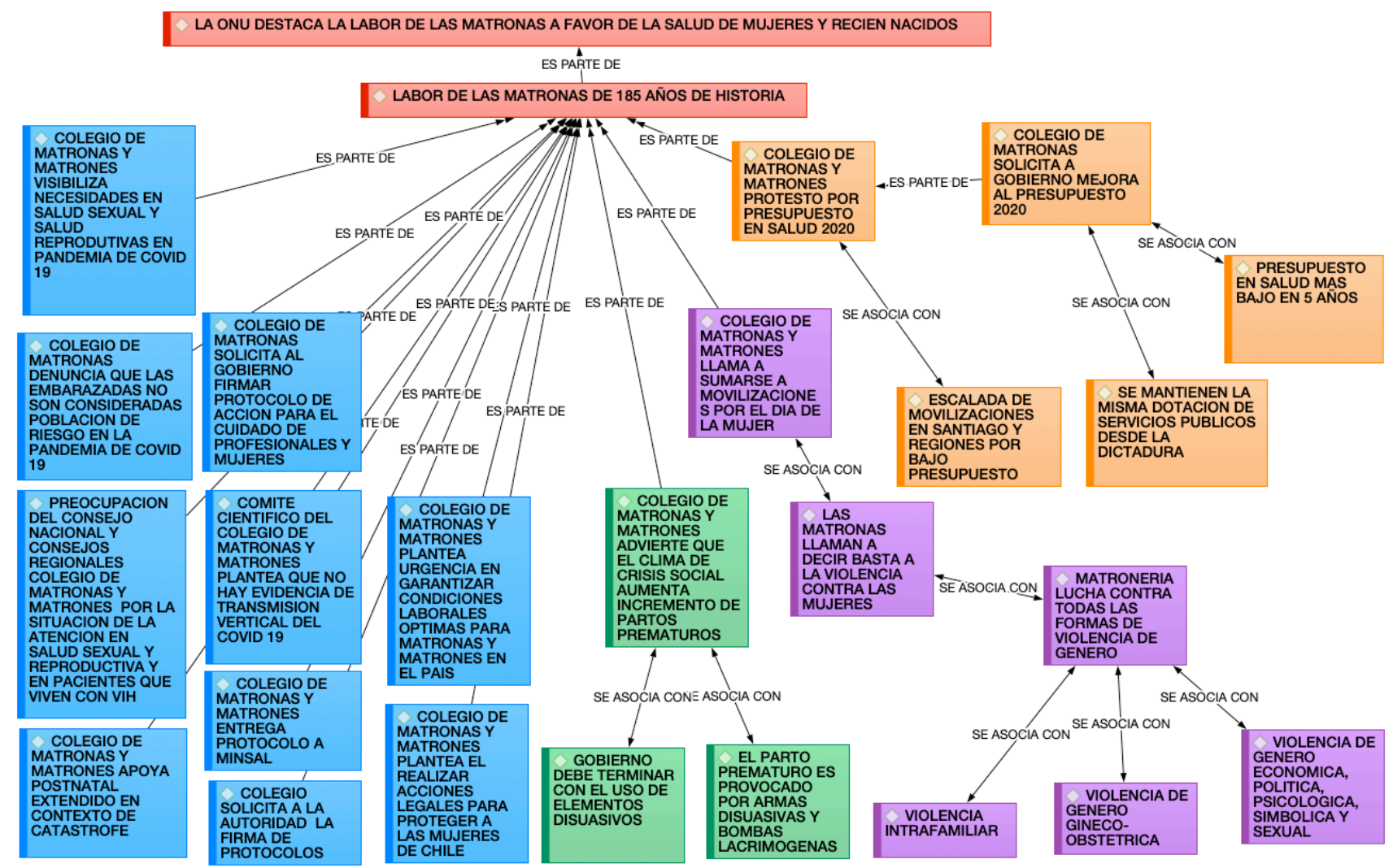

Fuente: Elaboración propia 
En relación con el «estallido social», se observa claramente que la matronería, como gremio, hace patente la molestia ante el presupuesto de salud para el año 2020, considerándolo insuficiente, recalcando que es el presupuesto más bajo de los últimos cinco años, que mantiene la deuda histórica del sistema de salud, fraguado en la dictadura, cuya dotación de servicios sanitarios en la actualidad es la misma, desde la década de los ochenta. Por consiguiente, el Colegio de Matronas y Matrones adhiere a las movilizaciones, junto a los demás gremios de la salud, en el contexto del «estallido social», en virtud de una demanda transversal, dada la precaria situación presupuestaria, lo cual está consignado en la noticia: «Colegio de Matronas pide al Gobierno que mejore el Presupuesto Salud 2020: "Estamos casi con la misma dotación de la dictadura"» (El Mostrador, 2019).

Siguiendo en el contexto del «estallido social», la noticia «Matronas llaman a participar de manifestaciones por el Día Internacional de la Mujer» (Radio Agricultura.cl, 2020), el gremio adhiere y convoca a las movilizaciones en torno a la conmemoración del 8 de marzo, Día Internacional de la Mujer, planteando que es imperativo terminar con la violencia contra las mujeres y que la matronería en Chile está comprometida en poner fin a variadas formas de violencia de género, sea esta económica, política, psicológica, simbólica y sexual, incluyendo también la violencia intrafamiliar y la violencia ginecoobstétrica.

En coherencia con lo anterior, el gremio advierte que, ante la crisis social presente, habrá un aumento de partos prematuros, debido al uso de armas disuasivas y bombas lacrimógenas, llamando al gobierno a terminar con la violencia generada por las fuerzas del orden y seguridad, según la nota periodística: «Matronas advierten que clima de crisis social podría generar partos prematuros» (Tele 13, 2019).

Algunas unidades textuales, que develan el accionar del Colegio de Matronas y Matrones, frente a la problemática de la SSySR en el contexto de pandemia son:

Voy a ser dura con mi juicio, pero de verdad, esto nos tiene muy mal: el Ministerio de Salud tiene a las mujeres abandonadas a su suerte. No es posible que llevemos más de dos meses exigiendo tener protocolos para atención en salud sexual y salud reproductiva en medio de esta pandemia y aún no tengamos nada, y no nos han dado ninguna respuesta» Anita Román, Presidenta del Colegio de Matronas y Matrones (El Periodista Online, 2020).

Todo el mundo sabe que deben vacunarse a los adultos mayores, embarazadas y niños pequeños, pero para esta pandemia las embarazadas no son 
consideradas población de riesgo, y eso es bastante curioso. Desde el día uno hemos estado solicitando que se testeé a todas las pacientes ingresadas a las maternidades. Ellas son pacientes de alto riesgo» Ximena González, Presidenta del Colegio de Matronas y Matrones Regional Rancagua (El Desconcierto, 2020).

Acorde con los anteriores enunciados, y en relación con la pandemia de COVID-19 y la crisis sanitaria generada, se puede observar que el Colegio de Matronas y Matrones toma un rol activo de denuncia, demanda y emplazamiento a la autoridad sanitaria y gobierno. En primer término, visibilizando las necesidades de SSySR, solicitando que las gestantes sean consideradas como una población prioritaria en la pandemia; requiriendo reiteradamente protocolos de acción, sin respuesta de la autoridad sanitaria, teniendo que desarrollar los equipos, de manera autónoma, protocolos propios, según lo descrito por Leiva et al. (2021); advirtiendo, dado el escenario, con interponer acciones legales para proteger a las mujeres del país. Por último, este gremio denuncia que las condiciones laborales en las que matronas y matrones realizan su trabajo no son óptimas debido a la exposición al contagio por COVID-19 y al olvido tácito de las gestantes y mujeres por parte del MINSAL y el gobierno.

\section{Limitaciones del estudio}

Se reconoce que la presente investigación es inicial y exploratoria, en tanto el fenómeno a analizar, siendo contingente, complejo y encontrándose aún en desarrollo, requiere de un acercamiento teórico y metodológico que permita dar cuenta de dicha complejidad; por ello, la aproximación del presente estudio es parcial, además de desafiante, ya que ha querido combinar el análisis documental de los medios de prensa con el análisis de entrevistas para dar cuenta de la producción de discursos por parte de matronas y matrones acerca de la crisis social y sanitaria que vive Chile.

\section{CONCLUSIONES}

La crisis social en Chile, performada en «estallido social» desde octubre de 2019, ha develado no solo las desigualdades sociales y económicas presentes en el país, sino que también ha dejado en evidencia el precario lugar de la salud como derecho, devenido bien de consumo y mercancía, en el escenario del modelo neoliberal. Por otra parte, la crisis sanitaria propiciada por el COVID-19, que ha suscitado problemáticas sociales, 
económicas y políticas a nivel global, para Chile no ha sido la excepción, agudizando la crisis del sector salud y agravando las desigualdades e inequidades preexistentes.

Desde el discurso de las matronas y matrones, se percibe una articulación entre crisis social y sanitaria, debido a que las y los profesionales de la matronería conviven cercanos a las necesidades de las mujeres y las comunidades. Por otro lado, se observa, en el relato, la tensión entre las necesidades en salud que deben satisfacer y la institucionalidad como barrera - en tanto que no provee los recursos necesarios para la atención sanitaria-, dando lugar a la autoprecarización profesional en el ejercicio del rol en el sistema de salud.

Se plantea, desde el discurso de la matronería, como parte de las demandas, la necesidad de una reforma estructural del sector salud en la que el rol del Estado debe cambiar, transformándose en un garante del derecho a la salud, cambiando las lógicas del mercado, disminuyendo las brechas de inequidad, fortaleciendo el sistema público de salud desde un enfoque de derechos y no como mercancía o bien de consumo. Estos planteamientos se articulan con las otras demandas sociales enunciadas en el «estallido social» del 18 de octubre.

El devenir histórico de la matronería, desde su creación y posterior tránsito de la mano con las transformaciones del sistema de salud chileno, ha mostrado una faceta que no es solo clínico-asistencial, en el cumplimiento del rol, sino que ha dado permanentes y sostenidas evidencias de un fuerte compromiso social, expresado en el accionar gremial y de las y los profesionales que ejercen la matronería. En la actualidad, de acuerdo con los hallazgos de esta investigación, se posicionan como agentes sociales y políticos de relevancia.

\section{AGRADECIMIENTOS}

Agradecemos a las y los colegas que contribuyeron con sus aportes y miradas, en tanto actores relevantes en momentos de crisis sanitarias y sociales, comprometidos con la salud sexual y salud reproductiva de las mujeres y sus familias, en cada rincón de Chile. 


\section{BIBLIOGRAFÍA}

BINFA, L., et al. Chilean midwives and midwifery students' views of women's midlife health-care needs. En: Midwifery. 2011, vol. 27, no. 4, pp. 417-423.

CASAS, L. Matronas piden al Gobierno no olvidar situación de embarazadas durante la pandemia. En: Biobiochile.cl. 2020. [Consulta: 9 junio 2020]. Disponible en: www.biobiochile.cl/noticias/nacional/chile/2020/04/10/matronas-piden-al-gobiernono-olvidar-situacion-de-embarazadas-durante-la-pandemia.shtml.

Castillo, C. A.; Molina, H. El Sistema de Salud chileno: análisis y perspectivas en el marco del estallido social de octubre de 2019. En: Revista Estado y Políticas Públicas. 2020, vol. 14, núm. 8, pp. 53-67.

Castillo, J. C. Colegio de Matronas pidió al Gobierno firmar protocolo de acción para el cuidado de los profesionales y las mujeres. En: ADN Radio.cl. 2020. [Consulta: 9 junio 2020]. Disponible en: www.adnradio.cl/nacional/2020/05/16/colegio-dematronas-pidio-al-gobierno-firmar-protocolo-de-accion-para-el-cuidado-de-losprofesionales-y-las-mujeres.html

CERDA, L. Formación profesional de la matrona/matrón en Chile: años de historia. En: Revista chilena de obstetricia y ginecología. 2020, vol. 85, núm. 2, pp. 115-122.

Cooperativa.CL. Seis matronas del Sótero del Río con Covid-19 tras contagio de funcionaria en culto evangélico. 2020. [Consulta: 9 junio 2020]. Disponible en: www.cooperativa.cl/noticias/sociedad/salud/coronavirus/seis-matronas-del-soterodel-rio-con-covid-19-tras-contagio-de/2020-04-14/143847.html

CReswell, J. W.; Poth, C. N. Qualitative inquiry and research design: Choosing among five traditions. Los Ángeles: SAGE Publications, 2018.

DenZIN, N.; LINCOLn, Y. The SAGE Handbook of Qualitative Research. Los Ángeles: SAGE Publications, 2017.

El DesConcierto. Colegio de Matronas: «Embarazadas no son consideradas población de riesgo en la pandemia». 2020. [Consulta: 9 junio 2020]. Disponible en: www.eldesconcierto.cl/2020/05/16/colegio-de-matronas-embarazadas-no-sonconsideradas-poblacion-de-riesgo-en-la-pandemia/

El Mostrador. Colegio de Matronas pide al Gobierno que mejore el Presupuesto Salud 2020: «Estamos casi con la misma dotación de la dictadura». 2019. [Consulta: 9 junio 2020]. Disponible en: www.elmostrador.cl/dia/2019/10/09/colegio-de-matronaspide-al-gobierno-que-mejore-el-presupuesto-salud-2020-estamos-casi-con-lamisma-dotacion-de-la-dictadura/

El Mostrador. Movimiento feminista sigue haciendo historia: dos millones de mujeres marcharon en Santiago y regiones en el 8M. 2020. Disponible en: www.elmostrador.cl/destacado/2020/03/08/movimiento-feminista-sigue-haciendohistoria-dos-millones-de-mujeres-marcharon-en-santiago-y-regiones-en-el-8m/ 
El Periodista Online. Matronas critican al Gobierno por primer fallecimiento de puérpera por Covid-19. 2020. [Consulta: 9 junio 2020]. Disponible en: www.elperiodista.cl/matronas-critican-al-gobierno-por-primer-fallecimiento-depuerpera-por-covid-19/

FUENTES-GARCÍA, A. El clamor de las inequidades: Estallido social y salud en Chile. En: Revista Chilena de Salud Pública. 2020, vol. 23, núm. 2, pp. 93-94.

Gattini, C. El sistema de salud en Chile. Observatorio chileno de Salud Pública, 2018. [Consulta: 11 junio 2020]. Disponible en: www.ochisap.cl

Giaconi Gandolfo. J. El Sistema de Salud chileno. En: ARS MEDICA. Revista de Ciencias Médicas. 2018, vol. 23, núm. 1.

Giaconi Gandolfo. J.; VAldivia C. G. El sistema de salud chileno. Ediciones Universidad Católica de Chile, 1994.

Giovanella, L., et al. 2021. ¿Es la atención primaria de salud integral parte de la respuesta a la pandemia de Covid-19 en Latinoamérica? En: Trabalho, Educação e Saúde. 2021, vol. 19.

GonzÁlez Moya, M.; ZÁrate CAmpos, M. S. Trabajo, miserias y recompensas: Asistentes sociales, enfermeras y matronas en la construcción de la política sanitaria chilena a mediados del siglo XX. En: Salud Colectiva. 2019, vol. 15.

Goyenechea, M.; Sinclaire, D. La privatización de la salud en Chile. En: Políticas Públicas Universidad de Santiago de Chile. 2013, vol. 6, núm. 1, pp. 35-52.

Homedes, N.; UGALde, A. Privatización de los servicios de salud: las experiencias de Chile y Costa Rica. En: Gaceta Sanitaria. 2002, vol. 16, núm. 1, pp. 54-62.

INFANTE-GRANDÓN, G. Claustrofobia territorial y sus consecuencias en el campo de la salud. En: Espacio Regional Revista de Estudios Sociales. 2017, vol. 2, núm. 14, pp. $39-47$.

InfANTE, G. N.; OBANDO, A. Horizontes para una matronería Comunitaria. Tensiones y oportunidades para una praxis territorial. En: Matronería actual. 2020, núm. 1.

Instituto Nacional De DeREChOS Humanos (INDH). INDH entrega nuevo reporte de cifras a cuatro meses de iniciada la crisis social. INDH, 2020. Disponible en: www.indh.cl/indh-entrega-nuevo-reporte-de-cifras-a-cuatro-meses-de-iniciada-lacrisis-social/

LABRA, M. E. La reinvención neoliberal de la inequidad en Chile: el caso de la salud. En: Cadernos de Saúde Pública. 2002, vol. 18, núm. 4, pp. 1041-1052.

LeIVA, G., et al. Protecting Women's and Newborns' Rights in a Public Maternity Unit During the COVID-19 Outbreak: The Case of Dra. Eloísa Díaz - La Florida Hospital in Santiago, Chile. En: Frontiers in Sociology. 2021, vol. 6. 
LiLlo, E., et al. Midwifery in Chile - A Successful Experience to Improve Women's Sexual and Reproductive Health: Facilitators \& Challenges. En: Journal of Asian Midwives. 2016, vol. 3, núm. 2, pp. 48-55.

LÓPEZ, P. Especificidades, prioridades y desafíos para el rol de la Matrona, Matrón, en contexto de pandemia por COVID-19. En: Matronería actual. 2020, vol. 1.

MARTínEZ-SALGAdO, C. El muestreo en investigación cualitativa: principios básicos y algunas controversias. En: Ciência \& Saúde Coletiva. 2012, vol. 17, núm. 3, pp. 613619.

MARZANO, M. Informed Consent, Deception, and Research Freedom in Qualitative Research. En: Qualitative Inquiry. 2007, vol. 13, núm. 3, pp. 417-436.

MinSAL. Ley 20120. Sobre la investigacion cientifica en el ser humano, su genoma, y prohibe la clonacion humana. 2006. Disponible en:

https://www.bcn.cl/leychile/navegar?idNorma $=253478$

MinSAL. Ley 20584. Regula los derechos y deberes que tienen las personas en relación con acciones vinculadas a su atención en salud. 2012. Disponible en: http://bcn.cl/2f7cj.

Minsal. Hitos de la Salud Chilena. Ministerio de Salud, 2016. Disponible en: http://web.minsal.cl/hitos-de-la-salud-chilena/

Minsal. Ministerio de Salud confirma primer caso de coronavirus en Chile. Ministerio de Salud, Gobierno de Chile, 2020. [Consulta: 13 junio 2020]. Disponible en: https://www.minsal.cl/ministerio-de-salud-confirma-primer-caso-de-coronavirusen-chile/

MoRÁN-FAUndÉS, J. M. Chile despertó: El modelo chileno, la matriz de desigualdad y la protesta de 2019. Crítica y Resistencias. En: Revista de conflictos sociales latinoamericanos. 2019, vol. 9, pp. 54-69.

NAVArro, F.; Tromben, C. «Estamos en guerra contra un enemigo poderoso, implacable»: los discursos de Sebastián Piñera y la revuelta popular en Chile. En: Literatura y Lingüística. 2019, vol. 40, pp. 295-324.

OlavarríA-GAMBI, M. Política de Salud en Chile. En: Revista venezolana de Gerencia. 2011, vol. 16, pp. 353-374.

Organización Mundial De La SAlud. Nuevo coronavirus 2019 (COVID-19). OMS, 2020. [Consulta: 6 agosto 2020]. Disponible en: www.who.int/es/emergencies/diseases/novel-coronavirus-2019/advice-for-public

PARADA-LezcAno, M., et al. Educación médica para la Atención Primaria de Salud: visión de los docentes y estudiantes. En: Revista Médica de Chile. 2016, pp. 10591066.

PÉREZ, G. Investigación Cualitativa. Retos e Interrogantes. Madrid: La Muralla, 1994, pp. 1-21. 
RAdio Agricultura.Cl. Matronas llaman a participar de manifestaciones por el Día Internacional de la Mujer. 2020. [Consulta: 9 junio 2020]. Disponible en: www.radioagricultura.cl/nacional/2020/03/07/matronas-1laman-a-participar-demanifestaciones-por-el-dia-internacional-de-la-mujer.html

RoJAS, K. La razones de la crisis estructural del sistema de salud público. En: El Mostrador. 2019. [Consulta: 9 junio 2020]. Disponible en:

www.elmostrador.cl/braga/2019/10/26/la-razones-de-la-crisis-estructural-delsistema-de-salud-publico/

RojAs, K. Los desafíos de la nueva matronería feminista y diversa. En: El Mostrador. 2020. [Consulta: 9 junio 2020]. Disponible en:

www.elmostrador.cl/braga/2020/05/05/los-desafios-de-la-nueva-matroneriafeminista-y-diversa/

Ruiz, C.; CAviedes, S. Estructura y conflicto social en la crisis del neoliberalismo avanzado chileno. En: ESPACIO ABIERTO Cuaderno Venezolano de Sociología. 2020, vol. 29, núm. 1, pp. 86-101.

Silva-PEÑA, I.; PAZ-MALdONADO, E. Formación docente para la justicia social desde la perspectiva emocional: Indagaciones narrativas en el contexto de la revolución del torniquete. En: Revista Interuniversitaria de Formación del Profesorado. 2019, pp. 195-212.

StAKe, R. E. Investigación con estudio de casos. Madrid: Ediciones Morata, 2007.

TELE 13. Matronas: Clima de crisis social podría generar partos prematuros. 2019. [Consulta: 9 junio 2020]. Disponible en: www.t13.cl/noticia/nacional/matronas-crisis-partos-prematuros

The Times CHILE. Colegio de Matronas pidió que el Gobierno apoye la extensión del postnatal. 2020. Disponible en: https://thetimes.cl/salud/colegio-de-matronas-pidioque-el-gobierno-apoye-la-extension-del-postnatal.shtml

Titelman, D. Reformas al sistema de salud en Chile: Desafios pendientes. Santiago, Chile:Unidad de Financiamiento para el desarrollo, 2000.

VERÓN, E. La semiosis social: fragmentos de una teoría de la discursividad. México: Editorial Gedisa, 1993.

VERÓN, E. La semiosis social, 2: ideas, momentos, interpretantes. Buenos Aires: Paidós, 2013.

\section{Material de Análisis según orden cronológico de octubre de 2019 a junio de 2020}

\section{Corpus de prensa escrita}

TELE 13. Matronas: Clima de crisis social podría generar partos prematuros. 2019. [Consulta: 9 junio 2020]. Disponible en:

https:/www.t13.cl/noticia/nacional/matronas-crisis-partos-prematuros 
El Mostrador. Colegio de Matronas pide al Gobierno que mejore el Presupuesto Salud 2020: «Estamos casi con la misma dotación de la dictadura». [Consulta: 9 junio 2020]. Disponible en: https://www.elmostrador.cl/dia/2019/10/09/colegio-de-matronas-pide-algobierno-que-mejore-el-presupuesto-salud-2020-estamos-casi-con-la-misma-dotacionde-la-dictadura/

RoJAS, K. La razones de la crisis estructural del sistema de salud público. El Mostrador. 2019. [Consulta: 9 junio 2020]. Disponible en:

https://www.elmostrador.cl/braga/2019/10/26/la-razones-de-la-crisis-estructural-delsistema-de-salud-publico/

RAdiO AgRicultura.Cl, Matronas llaman a participar de manifestaciones por el Día Internacional de la Mujer. 2020. [Consulta: 9 junio 2020]. Disponible en: https:/www.radioagricultura.cl/nacional/2020/03/07/matronas-llaman-a-participar-demanifestaciones-por-el-dia-internacional-de-la-mujer.html.

RoJAs, K. Los desafíos de la nueva matronería feminista y diversa. El Mostrador. 2020. [Consulta: 9 junio 2020]. Disponible en: https://www.elmostrador.cl/braga/2020/05/05/los-desafios-de-la-nueva-matroneriafeminista-y-diversa/

COOPERATIVA.CL. Seis matronas del Sótero del Río con Covid-19 tras contagio de funcionaria en culto evangélico. 2020. [Consulta: 9 junio 2020]. Disponible en: https:/www.cooperativa.cl/noticias/sociedad/salud/coronavirus/seis-matronas-del-soterodel-rio-con-covid-19-tras-contagio-de/2020-04-14/143847.html.

CASAS, L. Matronas piden al Gobierno no olvidar situación de embarazadas durante la pandemia. Biobiochile.cl. 2020. [Consulta: 9 junio 2020]. Disponible en: https://www.biobiochile.cl/noticias/nacional/chile/2020/04/10/matronas-piden-algobierno-no-olvidar-situacion-de-embarazadas-durante-la-pandemia.shtml

CAstillo, J. C. Colegio de Matronas pidió al Gobierno firmar protocolo de acción para el cuidado de los profesionales y las mujeres. ADN Radio.cl. 2020. [Consulta: 9 junio 2020]. Disponible en: https:/www.adnradio.cl/nacional/2020/05/16/colegio-de-matronas-pidioal-gobierno-firmar-protocolo-de-accion-para-el-cuidado-de-los-profesionales-y-lasmujeres.html

El DesCONCIERTO. Colegio de Matronas: «Embarazadas no son consideradas población de riesgo en la pandemia». 2020. [Consulta: 9 junio 2020]. Disponible en: https://www.eldesconcierto.cl/2020/05/16/colegio-de-matronas-embarazadas-no-sonconsideradas-poblacion-de-riesgo-en-la-pandemia/

El Periodista OnLINE. Matronas critican al Gobierno por primer fallecimiento de puérpera por Covid-19. 2020. [Consulta: 9 junio 2020]. Disponible en: https://www.elperiodista.cl/matronas-critican-al-gobierno-por-primer-fallecimiento-depuerpera-por-covid-19/ 